\title{
Automated Detection of Atrial Fibrillation using Fourier-Bessel Expansion and Teager Energy Operator from Electrocardiogram Signals
}

\author{
Shivnarayan Patidar ${ }^{1}$, Ashish Sharma ${ }^{1}$, Niranjan Garg ${ }^{2}$ \\ ${ }^{1}$ National Institute of Technology Goa, Ponda, India \\ 2 Nakshatra Heart and Multispeciality Hospital, Pipliyana Square, Indore, M.P., India
}

\begin{abstract}
This work presents a new method for detection of atrial fibrillation using predictors derived from Fourier-Bessel (FB) expansion and Teager energy operator (TEO) which are applied strategically on electrocardiogram (ECG) signals. The proposed method begins by extracting a set of direct and indirect predictors. The direct predictors are computed from pre-processed ECG signals themselves. A part of indirect predictors are computed from (a) RR-interval and heart rate (HR) signals, and (b) FB expansion along with its spectrum applied on $R R$ and HR signals. The rationale of using $F B$ expansion is that the clinical information is found to be more evident in the FB coefficients (FBC) and their spectrum than that of RR and HR signals themselves. In the same line of thought, TEO is applied on preprocessed ECG, RR-interval, HR signals, said FBC and their spectrum to obtain the other part of predictors. In all, 47 predictors are computed and subsequently they are fed to an ensemble system of bagged decision trees for classifying the ECG recordings. When evaluated with 2017 PhysioNet/CinC Challenge dataset (Phase II subset), the experimental outcomes demonstrate the $F_{1}$ scores of Normal, AF and other classes as: $90.89 \%, 80.07 \%, 72.24 \%$ respectively with overall $F_{1}$ score of $81 \%$ for the hidden test data.
\end{abstract}

\section{Introduction}

Atrial fibrillation (AF) is the most common cardiovascular disorder encountered in clinical practice [1]. Early and accurate diagnosis of AF can save many human lives. Conventionally, echocardiography and ECG tests in various forms are widely used to detect AF[2]. Echocardiography is quite expensive and needs experts in the field therefore its availability is limited to health care centers in urban areas. On the contrary, the ECG machine has shown to have huge clinical potential for affordable diagnosis of AF. However, manual interpretations of ECG recordings for detecting AF is not quite reliable [3]. Fortunately, the recent efforts in the field of signal processing, high performance computing and data mining techniques have paved the way for the emergence of automated diagnosis.

Currently, a wide variety of algorithms have been proposed for automatic detection of AF. These algorithms are mostly based on atrial activity and ventricular response analysis. The atrial activity analysis is based on the TQ interval of the ECG waveform. In presence of AF, the $\mathrm{P}$ wave are replaced by time varying fibrillatory f-waves. [4]. Popular atrial activity analysis based method to detect AF includes echo state neural network [5], Gaussian mixture model [4], average number of f-waves in a TQ interval [6], wavelet transform [7]. Most of these methods are prone to noise and therefore require high resolution ECG signals with lower noise contamination for accurate detection of $\mathrm{AF}[8]$.

On the other hand, detection of AF using ventricular response analysis is found to be more reliable and suitable for automatic, real-time AF detection [9]. Irregular and uncorrelated ventricular response in $\mathrm{AF}$ patients generate the irregular RR-intervals which serves as noise resistant measure for AF detection system. The popular ventricular response based methods include the use of Poincare plot [10] and Lorenz plot [11] to detect AF. Other methods use histograms [12,13], sample entropy, normalized fuzzy entropy, symbolic dynamics and Shannon entropy to detect AF [14-17].

\section{Methodology}

The proposed methodology for diagnosis of AF using ECG signals is presented in Figure 1. The details of main subsections are described as follows.

\subsection{Pre-processing}

In this work, one state-of-the-art is used to pre-process and detect the $\mathrm{R}$ peaks in ECG signals to obtain the RRinterval and HR signals [18]. 


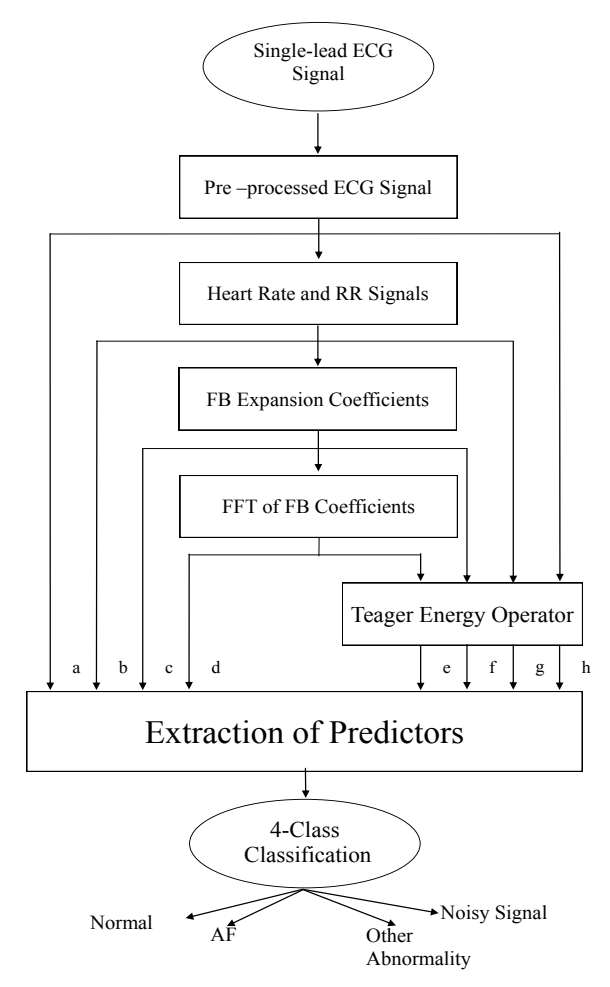

Figure 1. The proposed system.

\subsection{Applied Transform and Operation}

In context to signal processing, transforms and operators are commonly used for mapping the raw data from one domain to other which can be more informative. We have used FB expansion and TEO in this work which are described as follows:

\subsubsection{Fourier-Bessel Expansion}

A continuous-time signal $f(t)$, in the interval $(0,1)$, in terms of Bessel functions of first kind as basis functions can be expressed as follows [19]:

$$
f(t)=\sum_{i=1}^{M} A_{i} J_{0}\left(\lambda_{i} t\right)
$$

where, $A_{i}$ are the FB coefficients (FBC) which can be computed by using following equation:

$$
A_{i}=\frac{2 \int_{0}^{1} t f(t) J_{0}\left(\lambda_{i} t\right) d t}{\left[J_{1}\left(\lambda_{i}\right)\right]^{2}}
$$

where, $J_{0}(\cdot)$ and $J_{1}(\cdot)$ are the zero-order and first-order Bessel functions of first kind respectively. For $i=$ $1,2, \ldots, M$, the values of $\lambda_{i}$ are $M$ ascending order positive roots of $J_{0}(\lambda)=0$. The FBC $A_{i}$ are unique for a given signal.

\subsubsection{Teager Energy Operator}

TEO is a nonlinear function which is popularly used in the area of signal processing [20]. As against the conventional notion of energy that measure the sum of squared signal elements, TEO measures the energy of the system that generates it. The TEO for discrete-time signal $x[n]$ can be expressed as follows [20]:

$$
\operatorname{TE} O\{x[n]\}=x^{2}[n]-x[n-1] x[n+1]
$$

\subsection{Diagnostic Predictors}

In order to detect $\mathrm{AF}$, a set of useful predictors are derived from ECG signals, segmented ECG signals and the signals obtained after applying FB expansion and TEO. In this work, we have used the predictors as listed in Table 1 and 2. Some of these are: (a) Shannon entropy [21] (b) Sample Entropy [22] (c) Spectral Entropy [21] (d) Robust Permutation Entropy (RePE) [23] and (e) Morphological predictor: Inverse of normalized weighted difference of $\mathrm{P}$ and $\mathrm{T}$ peaks.

\subsection{Classification using an Ensemble Sys- tem of Bagged Decision Trees}

Ensemble classification [24] strategically generates a set of classifiers and combines them to get better performance than every one of them to address a particular machine learning problem. In an ensemble system of bagged decision tree, decision trees are used as the classification models and their decisions are combined by bootstrap aggregation [25]. For bagging with training set of size $N$, the decision trees are developed on the bootstrap replicas of the training dataset which are formed by randomly choosing $M$ observations out of $N$ with replacement, where $N$ is the training set size. The predictions of the individual classifiers are then combined using majority vote to cast the final decision.

\section{Results and Discussion}

The proposed work has been evaluated using 2017 PhysioNet/CinC Challenge dataset [18]. The training set contains 8,528 signals lasting from 9 to 61 seconds and the test set contains 3,658 signals of similar lengths (and class distributions). After adequate pre-processing, predictors are derived from FB expansion and TEO which in turn are applied strategically on ECG signals. Figure 1 shows the predictor extraction workflow. A set of carefully chosen direct and indirect predictors as listed in Tables are extracted. Where in the Table 2, Md, Mo, Ku, and $S k$ represent median, mode,kurtosis and skewness respectively. The direct predictors are computed from pre-processed ECG signals 
as depicted in the Figure 1 with arrow marked by (a). A part of the indirect predictors are computed from (i) RR and HR signals, (ii) FB expansion along with its spectrum as marked by (b), (c) and (d) respectively . In order to obtain the other part of predictors, we have applied TEO on the above set of signals obtained at different levels of proposed framework as marked by (e)-(h). We have found 47 clinically significant predictors as listed in Tables that ultimately yielded robust 10 -cross validation based results.

Table 1. List of predictors used for classification

\begin{tabular}{|l|l|l|l|l|}
\hline $\begin{array}{l}\text { S. } \\
\text { No. }\end{array}$ & Predictors & \multicolumn{3}{|c|}{ Input Signals } \\
\hline & & ECG & HR/RR & $\begin{array}{l}\text { FBC- } \\
\text { HR/FBC- } \\
\text { RR }\end{array}$ \\
& & & & $/ 12$ \\
\hline 1 & Sample Entropy & 34,35 & $10 / 11$ & 19 \\
2 & Spectral entropy & 21 & 16,7 & 8,9 \\
3 & RePE & 5 & & $/ 1$ \\
4 & Shannon entropy & 22 & $/ 2,3,4$ & \\
5 & Shannon entropy of TEO & & 13 & \\
6 & Energy of TEO & & & \\
\hline
\end{tabular}

Table 2. List of predictors used for classification

\begin{tabular}{|c|c|c|c|c|c|c|c|c|}
\hline S. & Input Parameters & \multicolumn{7}{|c|}{ Predictors } \\
\hline & & $\mu$ & $M d$ & Mo & $\sigma$ & $\sigma^{2}$ & $K u$ & $S k$ \\
\hline 1 & HR/RR Signals & 27 & $28 /$ & $29 /$ & & 30 & 1 & $l_{26}$ \\
\hline 2 & FBC of HR & & & 20 & & & & \\
\hline 3 & TEO of HR & & & & 18 & & 14 & \\
\hline 4 & TEO of ECG & & & & & & 15 & \\
\hline 5 & TEO of FBC of $\mathrm{HR}$ & & & & 17 & & & 16 \\
\hline 6 & Kurtosis of ECG & & 31 & & & & & 32 \\
\hline 7 & Median of ECG & & 33 & & & & & \\
\hline 8 & Widths of $\mathrm{R}$ peaks & & & 37 & 39 & 41 & & \\
\hline 9 & Widths of S peaks & & & 38 & & 42 & & \\
\hline 10 & $\begin{array}{l}\text { Prominences of } R \\
\text { peaks }\end{array}$ & & & & 40 & & & \\
\hline 11 & $\begin{array}{l}\text { Ratios of widths of } R \\
\& S \text { peaks }\end{array}$ & 36 & & & & & & \\
\hline 12 & Diff. of $R \& S$ peaks & 43 & 45 & & & & & \\
\hline 13 & $\begin{array}{l}\text { Diff. of widths of } R \\
\& S \text { peaks }\end{array}$ & 44 & & & & & & \\
\hline 14 & $\begin{array}{l}\text { Diff. of locations of } \\
R \& S \text { peaks }\end{array}$ & & & & & & 46 & \\
\hline
\end{tabular}

Using the auto-search engine in Matlab, an optimal ensemble classifier model based on the bootstrap aggregation is generated for classifying the ECG recordings into following classes: normal, AF, others and noisy.

A graph to illustrate the importance of each predictors used in decision making for the ensemble classifier is shown in Figure 2. It indicates the relative relevance of a set of 47 predictors. The out-of-bag (OOB) classification error as a function of the number of decision trees were used to construct the ensemble as illustrated in Figure 3. The optimal ensemble classifier was constructed by using
477 weak learners that are decision trees with minimum leaf size of 2 and with training instances of 8528 .

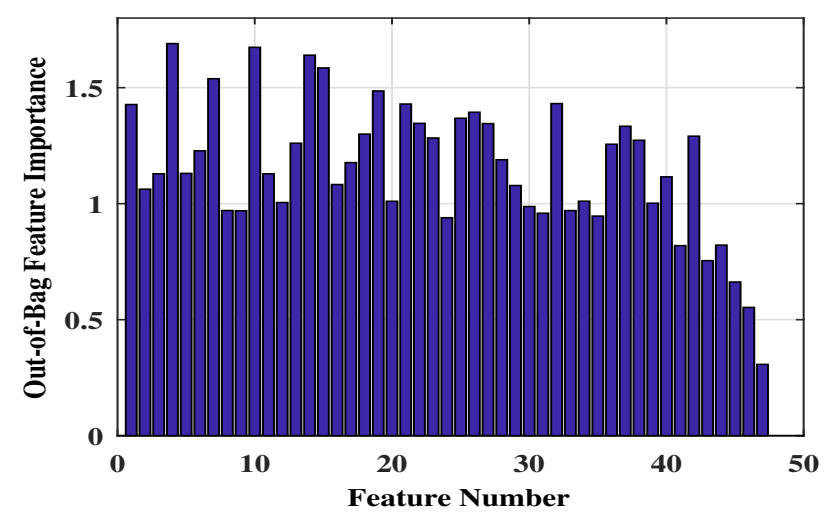

Figure 2. The predictor's importance.

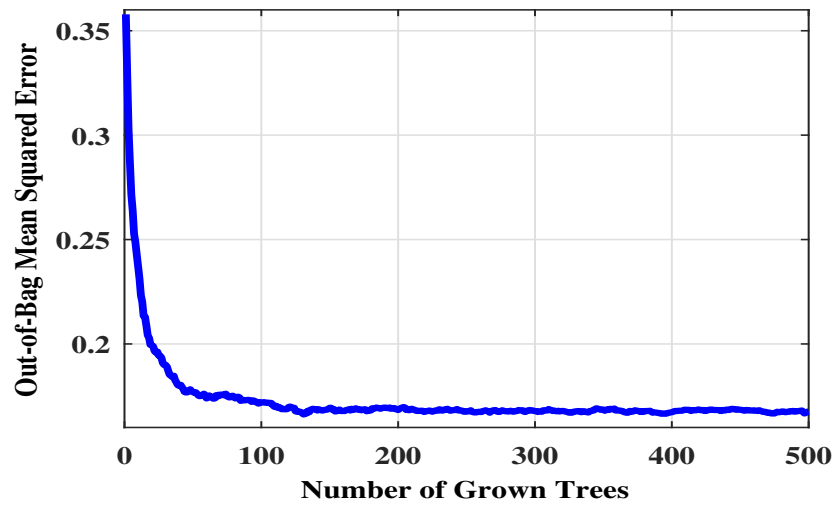

Figure 3. OOB classification error versus weak learners.

We have obtained the average $F_{1}$ scores for normal rhythm, AF, other rhythm and noisy as follows: $90 \%, 77 \%$, $73 \%, 61 \%$ with overall average $F_{1}$ score of $80 \%$ for first three classes with training data applying 10-cross validation. The average accuracy of $84 \%$ is obtained. The test $F_{1}$ scores of Normal, AF, other classes and noisy recordings using hidden data from PhysioNet (Phase II subset) are as follows: $90.89 \%, 80.07 \%, 72.24 \%$ and $50.07 \%$ respectively with overall $F_{1}$ score of $81 \%$ for the first three classes. This work reveals that the predictors derived using FB expansion and TEO are quite promising for better characterization of different ECG signals to detect and identify $\mathrm{AF}$ and other cardiovascular disorder (CVDs).

\section{Conclusion}

In this work, we have explored the strength and applicability of FB expansion and TEO based algorithm for 
automated diagnosis of AF. Our method has shown significant performance on a large and diverse dataset with noisy records. This work has clinical potential to be realized into an automatic real-time system for detection of $\mathrm{AF}$ and other CVDs.

\section{Acknowledgements}

We acknowledge the financial support received from (a) NIT Goa, Seed money project entitled "Primary level screening of common heart disorders using non-invasively measured heart sound signals" and (b) DST India, ECR project entitled "Analysis of cardiovascular disorders using heart sound signals", project no. ECR/2017/000062.

\section{References}

[1] Potter BJ, Le Lorier J. Taking the pulse of atrial fibrillation. The Lancet 2015;386(9989):113-115.

[2] Patidar S, Pachori RB, Acharya UR. Automated diagnosis of coronary artery disease using tunable-Q wavelet transform applied on heart rate signals. Knowledge Based Systems 2015;82:1-10.

[3] Silber EN, Katz LN. Heart Disease. MacMillan Publishing Co., New York, 1982.

[4] Ladavich S, Ghoraani B. Rate-independent detection of atrial fibrillation by statistical modeling of atrial activity. Biomedical Signal Processing and Control 2015;18:274281.

[5] Petrenas A, Marozas V, Sornmo L, Lukosevicius A. An echo state neural network for qrst cancellation during atrial fibrillation. IEEE Transactions on Biomedical Engineering 2012;59(10):2950-2957.

[6] Du X, Rao N, Qian M, Liu D, Li J, Feng W, Yin L, Chen $\mathrm{X}$. A novel method for real-time atrial fibrillation detection in electrocardiograms using multiple parameters. Annals of Noninvasive Electrocardiology 2014;19(3):217-225.

[7] García M, Ródenas J, Alcaraz R, Rieta JJ. Application of the relative wavelet energy to heart rate independent detection of atrial fibrillation. Computer Methods and Programs in Biomedicine 2016;131:157-168.

[8] Colloca R. Implementation and testing of atrial fibrillation detectors for a mobile phone application 2013;

[9] Carrara M, Carozzi L, Moss TJ, de Pasquale M, Cerutti S, Ferrario M, Lake DE, Moorman JR. Heart rate dynamics distinguish among atrial fibrillation, normal sinus rhythm and sinus rhythm with frequent ectopy. Physiological measurement 2015;36(9):1873-1888.

[10] Park J, Lee S, Jeon M. Atrial fibrillation detection by heart rate variability in poincare plot. Biomedical Engineering online 2009;8(1):38.

[11] Sarkar S, Ritscher D, Mehra R. A detector for a chronic implantable atrial tachyarrhythmia monitor. IEEE Transactions on Biomedical Engineering 2008;55(3):1219-1224.

[12] Tateno K, Glass L. Automatic detection of atrial fibrillation using the coefficient of variation and density histograms of rr and $\delta \mathrm{rr}$ intervals. Medical and Biological Engineering and Computing 2001;39(6):664-671.

[13] Huang C, Ye S, Chen H, Li D, He F, Tu Y. A novel method for detection of the transition between atrial fibrillation and sinus rhythm. IEEE Transactions on Biomedical Engineering 2011;58(4):1113-1119.

[14] Alcaraz R, Abásolo D, Hornero R, Rieta JJ. Optimal parameters study for sample entropy-based atrial fibrillation organization analysis. Computer Methods and Programs in Biomedicine 2010;99(1):124-132.

[15] Lake DE, Moorman JR. Accurate estimation of entropy in very short physiological time series: the problem of atrial fibrillation detection in implanted ventricular devices. American Journal of Physiology Heart and Circulatory Physiology 2011;300(1):H319-H325.

[16] DeMazumder D, Lake DE, Cheng A, Moss TJ, Guallar E, Weiss RG, Jones S, Tomaselli GF, Moorman JR. Dynamic analysis of cardiac rhythms for discriminating atrial fibrillation from lethal ventricular arrhythmias. Circulation Arrhythmia and Electrophysiology 2013;6:555-561.

[17] Zhou X, Ding H, Ung B, Pickwell-MacPherson E, Zhang Y. Automatic online detection of atrial fibrillation based on symbolic dynamics and shannon entropy. Biomedical Engineering Online 2014;13(1):18.

[18] Clifford G, Liu C, Moody B, Lehman L, Silva I, Li Q, Johnson A, Mark R. Af classification from a short single lead ecg recording: The physionet computing in cardiology challenge 2017. In Computing in Cardiology. IEEE, 2017;

[19] Schroeder J. Signal processing via fourier-bessel series expansion. Digital Signal Processing 1993;3(2):112-124.

[20] Kaiser JF. Some useful properties of teagers energy operators. In IEEE international conference on acoustics, speech, and signal processing, ICASSP-93. IEEE, 1993; 3.

[21] Shannon C. A mathematical theory of communication. The Bell System Technical Journal 1948;27 (3):379423.

[22] Richman JS, Moorman JR. Physiological time-series analysis using approximate entropy and sample entropy. American Journal of Physiology Heart and Circulatory Physiology 2000;278(6):H2039-H2049.

[23] Keller K, Unakafov AM, Unakafova VA. Ordinal patterns, entropy, and eeg. Entropy 2014;16(12):6212-6239.

[24] Polikar R. Ensemble based systems in decision making. IEEE Circuits and Systems Magazine 2006;6(3):21-45.

[25] Breiman L. Bagging predictors. Machine Learning 1996; 24(2):123-140.

Address for correspondence:

Dr Shivnarayan Patidar

Department of Electronics and Communication Engineering

National Institute of Technology Goa, Ponda

India, 403401

shivnarayan.patidar@nitgoa.ac.in 